\title{
Some limitations on the Premack principle
}

\author{
WILLIAM L. CROLL \\ Carleton University, Ottawa, Ontario K1S 5B6, Canada
}

\begin{abstract}
Existing data show that the Premack principle is wrong in several regards: (a) the sufficient condition for reinforcement is not a difference in preference, (b) magnitude of reinforcement is not an increasing function of the preference difference between the instrumental and contingent activities, and (c) preference can not be adequately measured in terms of response probability.
\end{abstract}

Premack (e.g., 1965, 1971) had demonstrated a number of weaknesses in traditional reinforcement theories and has proposed a relational theory designed to avoid these difficulties. The major difference between the relational theory and traditional theories is in the conditions considered to be sufficient for the occurrence of reinforcement. Traditional theories assumed (either explicitly or implicitly) that activities are negative, neutral, or positive; and that only neutral activities function as instrumental responses, while only positive activities function as reinforcers. Thus, reinforcement should occur only when a positive activity is made contingent upon a neutral activity. The relational theory considers the negative-neutral-positive trichotomy to be irrelevant to reinforcement. Instead, it assumes that all activities are ordered along a preference continuum and that the sufficient condition for reinforcement is merely a difference in preference. Thus, the relational theory does not require that instrumental responses be neutral nor that reinforcers be positive: all that is required is that the reinforcer be preferred over the instrumental response.

Numerous studies have shown that the predictions of the relational theory are sometimes more accurate than are those of traditional theories. Activities which were traditionally considered to be neutral do sometimes function as reinforcers, and activities which were traditionally considered to be positive do sometimes function as instrumental responses (e.g., Premack, 1971). There are, however, several well established results contradicting the relational theory that have gone unnoticed. Together they show that magnitude of reinforcement is not an increasing function of preference difference, and that preference difference is not the sufficient condition for reinforcement. They also provide additional evidence that the response probability measure of preference which has so often been used in conjunction with the relational theory does not provide an adequate measure of preference.

The results contradicting the relational theory come from comparisons among reinforcement contingencies which have a common positive contingent response and instrumental responses of different degrees of aversiveness. In such situations, the preference difference between the instrumental and contingent responses increases as the instrumental response becomes more aversive. Since the theory assumes that reinforcement magnitude is an increasing function of preference difference, it thus predicts Ss will perform a highly aversive activity more than a moderately aversive activity in order to obtain a given goal. This prediction is counter to common sense and is contradicted by numerous studies. For example, demand curves from economic research show that the rate at which a given commodity is purchased decreases as the price of that commodity increases, which is opposite the relational theory's prediction (e.g., Stigler, 1952, p. 58). Similarly, in research on gambling, the likelihood of accepting a given gamble decreases as the bet required to play increases (e.g., Tversky, 1967). In the limit, such results also show that preference difference is not the sufficient condition for reinforcement. As just noted, the theory predicts that reinforcement magnitude increases without bound as the instrumental response becomes more aversive. In actuality, just the opposite happens: As the instrumental activity becomes more aversive, a point will be reached where reinforcement fails to occur (e.g., Olds \& Sinclair, 1957). The fact that such failures occur in the presence of a substantial preference difference indicates that preference difference is not the sufficient condition for reinforcement.

Although the above results clearly indicate that the relational theory does not accurately identify the sufficient condition for reinforcement nor the function describing reinforcement magnitude, they unfortunately do not reveal the true sufficient condition and reinforcement magnitude function. They do, however, reveal an important weakness in prior attempts to obtain this information. In most of these attempts, preference has been measured in terms of response probability, i.e., the proportion of time $S$ spends engaged in an activity when it is freely available. Two weaknesses of this procedure have already been discussed in the literature. Hilgard and Bower (1966, p. 486) observed that response rate can be directly conditioned, which makes it a questionable measure of preference. Similarly, Kaess and Weir (1968) found that response probability measures of preference sometimes do not correlate highly with choice measures of preference. Since many investigators feel that choice is perhaps the most accurate measure of preference, any measure which fails to correlate highly with choice is suspect. The results 
considered in the present paper suggest still another weakness in the response probability measure. Recall that failures of the relational theory occur when the instrumental activity is aversive. The response probability measure obscures these failures by failing to discriminate among activities of different degrees of aversiveness, assigning a value of zero to all aversive activities. It seems likely that it is this collapsing of the entire negative region of the preference continuum by the response probability measure which has led to neglect of aversive activities and hence to misidentification of the sufficient condition for reinforcement and the reinforcement magnitude function. In any event, it is clear that correct identification can not occur in the absence of a preference measure which discriminates degrees of aversiveness. Consequently, the development of an adequate preference measure appears to be the most important problem at the present time.

Several writers have suggested that the best way of measuring preference is by paired comparison choices, arguing that it is intuitively obvious that Ss tend to choose those alternatives they prefer most (Greeno, 1968, p. 40; Hilgard \& Bower, 1966, p. 486). Although the paired-comparison method may accurately reveal rank orders of preference, and although it is successful in discriminating among activities of different degrees of aversiveness, Haaf (1971) has noted that is does not identify the zero or neutral point on the preference continuum. Consequently, it does not show which activities are aversive, which are neutral, and which are positive; thus is can not be used to determine the sufficient condition for reinforcement and the reinforcement magnitude function. Haaf suggested that a more complicated paired-comparison procedure might successfully yield the required zero point. Letting $\mathrm{x}+\mathrm{y}$ represent a bundle consisting of commodities $\mathrm{x}$ and $\mathrm{y}$, this procedure requires $S$ to make comparisons of the form $\mathrm{x} v \mathrm{x}+\mathrm{y}$ and $\mathrm{y} v \mathrm{x}+\mathrm{y}$ in addition to the standard $x$ vs $y$ comparisons. The zero point is then defined as the point with respect to which the sum of two single-object values equals the value of the corresponding composite bundle. Although this procedure may prove to be useful, it has not yet been shown that the zero point yielded by this procedure does in fact represent affective neutrality (Croll, 1973). Thus, while the procedure seems promising, it is not possible at the present time to determine whether it can be used to find the sufficient condition for reinforcement and the reinforcement magnitude function.

An alternative preference measure that has apparently not been investigated consists of obtaining preference ratings by the method of successive categories. With this method, $\mathrm{S}$ is required to rate each activity in terms of a scale on which zero represents neutrality, positive scores represent different degrees of appetitiveness, and negative scores represent different degrees of aversiveness. In addition to being more economical than the paired-comparison procedure, the method of successive categories does identify the zero point and does discriminate among degrees of aversiveness. These considerations clearly recommend the use of the method of successive categories, but there are two arguments which might be invoked against its use. Greeno (1968, p. 45) has argued that is does not provide sufficient information to permit precise quantitative predictions. This objection, however, holds only when the procedure is used within the context of Luce's (1959) theory of choice and does not represent a general weakness of the procedure. The second possible objection is that the procedure can be used only with articulate human Ss, limiting the generality of any relationships that may be discovered through its use. This is a real problem, but there is some evidence that it can be solved. Bock and Jones (1968, p. 213) have noted that results obtained with the method of successive categories can frequently be used to predict results obtained with the method of paired comparisons. This suggests that it may be possible to determine the sufficient condition for reinforcement and the reinforcement magnitude function with adults and the method of successive categories, then extend these results to children and infrahumans using the method of paired comparisons. This approach, and the approach discussed by Haaf, appear to be the most promising at the present time.

\section{REFERENCES}

Bock, R. D., \& Jones, L. V. The measurement and prediction of judgment and choice. San Francisco: Holden-Day, 1968.

Croll, W. L. The rational zero point and reinforcement. Bulletin of the Psychonomic Society, 1973, 1, 431-432.

Greeno, J. G. Elementary theoretical psychology. Reading, Massachusetts: Addison-Wesley, 1968.

Haaf, R. A. The rational zero point on incentive-object preference scales: A developmental study. Developmental Psychology, 1971, 5, 537.

Hilgard, E. R., \& Bower, G. H. Theories of learning. (3rd ed.) New York: Appleton-Century-Crofts, 1966

Kaess, D. W., \& Weir, M. W. Response technique as a factor in determining children's preference for complexity. Psychonomic Science, 1968, 11, 367-368.

Luce, R. D. Individual choice behavior: A theoretical analysis. New York: Wiley, 1959.

Olds, J., \& Sinclair, J. C. Self-stimulation in the obstruction box. American Psychologist, 1957, 12, 464.

Premack, D. Reinforcement theory. In D. Levine (Ed.), Nebrasks symposium on motivation 1965. Lincoln: University of Nebraska Press, 1965. Pp. 123-180.

Premack, D. Catching up with common sense or two sides of a generalization: Reinforcement and punishment. In R. Glaser (Ed.), The nature of reinforcement. New York: Academic Press, 1971. Pp. 121-150.

Stigler, G. J. The theory of price. New York: MacMillan, 1952. Tversky, A. Utility theory and additivity analysis of risky choices. Journal of Experimental Psychology, 1967, 75, 27-37.

(Received for publication March 4, 1974.) 